nephron

Practice
Nephron 2017;137:268-272

DOI: $10.1159 / 000476079$
Received: April 19, 2017

Accepted after revision: April 24, 2017

Published online: June 15, 2017

\title{
Drug Discovery to Halt the Progression of Acute Kidney Injury to Chronic Kidney Disease: A Case for Phenotypic Drug Discovery in Acute Kidney Injury
}

\author{
Neil Hukriede ${ }^{a, b} \quad$ Andreas Vogt ${ }^{c, d}$ Mark de Caestecker ${ }^{e}$ \\ a Department of Developmental Biology, ${ }^{b}$ Center for Critical Care Nephrology, ${ }^{\mathrm{c} D e p a r t m e n t}$ of Computational \\ and Systems Biology, and d Drug Discovery Institute, University of Pittsburgh, Pittsburgh, PA, and e Department of \\ Medicine, Vanderbilt University Medical Center, Nashville, TN, USA
}

\section{Keywords}

Drug discovery · Phenotypic screening · Molecular targets .

Zebrafish · Acute kidney injury

\begin{abstract}
The cellular responses that occur following acute kidney injury (AKI) are complex and dynamic, involving multiple cells types and molecular pathways. For this reason, early selection of defined molecular targets for therapeutic intervention is unlikely to be effective in complex in vivo models of $\mathrm{AKI}$, let alone Phase 3 clinical trials in patients with even more complex AKI pathobiology. Phenotypic screening using zebrafish provides an attractive alternative that does not require prior knowledge of molecular targets and may identify compounds that modify multiple targets that might be missed in more traditional target-based screens. In this review, we discuss results of an academic drug discovery campaign that used zebrafish as a primary screening tool to discover compounds with favorable absorption, metabolism, and toxicity that enhance repair when given late after injury in multiple models of AKI. We discuss how this screening campaign is being integrated into a more comprehensive, phenotypic, and target-based screen for lead compound optimization.

(c) 2017 S. Karger AG, Basel
\end{abstract}

\section{Background}

Patients with acute kidney injury (AKI) are at an increased risk of contracting progressive chronic kidney disease (CKD) [1-4], but no therapies have been proven to reduce post-AKI CKD progression [5]. One of the main reasons for this is that patients often present late in the clinical course of AKI, and many of the drugs that have been evaluated in clinical trials, were only effective in experimental models of AKI when given before the initiating injury [6]. As an academic drug discovery program, we sought to address this limitation by focusing our therapeutic discovery on the cellular repair mechanisms that are generally activated after the initiating injury has occurred [7].

Contribution from the AKI and CRRT 2017 Symposium at the 22nd International Conference on Advances in Critical Care Nephrology, Manchester Grand Hyatt, San Diego, Calif., USA, March 7-10, 2017. This symposium was supported in part by the NIDDK funded University of Alabama at Birmingham-University of California San Diego O'Brien Center for Acute Kidney Injury Research (P30 DK079337).

\section{KARGER}

(c) 2017 S. Karger AG, Basel

E-Mail karger@karger.com

www.karger.com/nef
Dr. Mark de Caestecker

Nephology Division

Vanderbilt University Medical Center, S3223, Medical Center North

116121 Street Avenue South Nashville, TN 37232 (USA)

E-Mail mark.de.caestecker@ vanderbilt.edu 


\section{Phenotypic vs. Target-Based Drug Discovery for AKI}

Most of the post-genomics era drug-discovery efforts have been target based focusing initially on the identification of compounds that modify selected molecular targets in vitro. However, the majority of first-in-class drugs approved by the FDA were discovered in phenotypic, not target-based, screens [8]. This is relevant for AKI drug discovery where multiple cells types and molecular pathways are involved in regulating injury and repair, so the selection of a single molecular target early in a drug discovery campaign may not survive efficacy studies in complex in vivo models. In addition, since access to kidney biopsies from patients with AKI is limited [6,9], very little is known about the molecular mechanisms of injury and repair in humans, so that the likely impact of these therapeutic interventions in patients with AKI will be essentially unknown until results of hypothesis testing Phase 3 clinical trials become available.

With the development of new instrumentation and imaging technologies, there has been increased interest, particularly in academic drug-discovery programs, in the use of invertebrate (C. elegans and Drosophila) and vertebrate (zebrafish) models as primary tools for high-content screens (HCS) [10-12]. These platforms have 2 major advantages over target-based discovery: (a) phenotypic screens do not require prior knowledge of the molecular target, and may identify compounds that simultaneously engage multiple targets. This may be important in the complex milieu of the injured kidney and might be missed in traditional target-based screens; and (b) phenotypic screens provide insight into absorption, distribution, metabolism, excretion and toxicity (ADMET) at an early stage in drug development. This has the potential to accelerate drug development by allowing for more rapid transition to animal models with minimal requirement for ADMET optimization.

\section{The Use of Zebrafish for Phenotypic Drug Discovery in Kidney Disease}

Zebrafish have genetic conservation with humans and are genetically malleable, so they can be used to screen for compounds that correct mutation-associated phenotypes [13]. Zebrafish are also amenable to HCS: (a) screening can be performed using fertilized eggs ( 400 per clutch) over the first $72 \mathrm{~h}$ post fertilization (hpf), since most larval organs, including kidneys, liver, and the cardiovascular system are functional by 24-48 hpf [14]; (b) eggs and lar-

Drug Discovery to Halt the Progression of AKI to CKD vae are small enough to array in multi-well platforms; can be isolated and dispensed using microfluidic technologies; and can be soaked in robotically dispensed compounds [13]; (c) zebrafish are transparent and this aspect can be exploited for analysis of internal organs, and for analysis of signaling pathways or cells using fluorescent reporters [15]. In addition, the zebrafish larval kidney, while simple, has anatomical features of the mammalian kidney that are not conserved in invertebrate models. This includes a glomerular filtration system linked to pulsatile blood flow, and renal segmentation that conserves many of the functions and markers found in the mammalian kidney [16]. These features make zebrafish larvae an ideal model for phenotypic, kidney-related drug discovery [17].

\section{Screening for Small Molecules that Enhance Repair after AKI}

Our goal was to identify molecules that enhance repair after AKI, so we developed proliferation-based assays associated with expansion of renal progenitor cells in embryonic and larval zebrafish. Our working hypothesis was that compounds that promote the expansion of renal progenitor cells would also enhance proliferative repair after AKI. We screened $~ 2,000$ compounds to identify larvae that developed pericardial edema, a sign of abnormal kidney function associated with impaired water excretion. This was followed by an in situ hybridization screen to look for expansion of $L h x 1$ expressing renal progenitor cells. Using this approach we identified 4-phenylthiobutanoic acid (PTBA), a histone deacetylase (HDAC) inhibitor that caused proliferation-dependent expansion of renal progenitor cells [18]. To determine whether PTBA also enhanced repair after AKI, we initially exploited a model of gentamicin-induced AKI in zebrafish larvae in which intra-cardiac injection of gentamicin causes AKI with renal tubular cell death followed by proliferative repair [19]. There was increased survival of larvae soaked in the PTBA pro-drug, methyl4-phenylbutanoate, M4PTB/UPHD25, after gentamicin-induced AKI. This was associated with a marked increase in renal tubular cell proliferation [20]. We next evaluated the effects of UPHD25 in a mouse model of ischemia reperfusion-induced AKI (IR-AKI) [21]. UPHD25 treatment initiated $24 \mathrm{~h}$ after injury reduced post-injury renal fibrosis and was associated with increased effective renal tubular cell proliferation [20]. UPHD25 initiated 4 days after injury also reduced renal 
fibrosis and enhanced proliferative repair of renal tubular epithelial cells in a mouse model of aristolochic acidinduced AKI [22].

\section{Automated Image-Based Zebrafish Embryonic Kidney Progenitor Cells Assays}

Encouraged by the success of our initial screen, we developed an automated zebrafish embryonic kidney progenitor cell (EKPC) assay for PTBA analog optimization. For this, we applied an artificial intelligence-based imaging technique called the cognitive network technology, which quantifies fluorescent signals associated with fluorescent markers in live zebrafish larvae [23]. We used 2 transgenic zebrafish: (a) the $L h \times 1$ fluorescent reporter, $\operatorname{Tg}(\operatorname{lh} x 1 a: E G F P)$, to quantify renal progenitor cell numbers [24]; and(b)theCadherin 17reporter, $\operatorname{Tg}(c d h 17: E G F P)$, to quantify the expansion of renal tubular epithelium [25]. Using these, we developed dose-response curves for PTBA analogs (Fig. 1) [24, 25], and found that 20 out of 64 analogs were active with EC50s $<3 \mu \mathrm{M}$ [25]. Of the 6 active compounds (including PTBA) with modifications to acid functionality, 4 increased survival in zebrafish AKI assays and of these, the PTBA pro-drugs, UPHD25 and UPHD186, reduced fibrosis after IR-AKI in mice (online suppl. Fig. 1; for all online suppl. material, see www.karger. com/doi/10.1159/000476079). Furthermore, UPHD186 (but not UPHD25), reduced fibrosis after unilateral ureteric obstruction, and reduced post-injury renal fibrosis when treatment was delayed 3 days after IR-AKI [25]. In addition, treatment with UPHD186 for 7 days at up to $5 \times$ the effective therapeutic dose, showed no demonstrable toxicity in mice (unpublished data). These findings indicate that our screening platform can identify nontoxic compounds that enhance proliferative repair in multiple mouse models of AKI, even when treatment is delayed days after the initiating injury. This provides the potential

Fig. 1. Cognition network technology (CNT) image-based analysis of EGFP-labeled transgenes. a Upper panel: $\operatorname{Tg}(\operatorname{lh} x 1 \mathrm{a}: E G F P)^{p t 303}$ treated at $3 \mathrm{hpf}$ with vehicle (0.5\% DMSO) or UPHD25 (1.5 $\mu \mathrm{M})$. Images acquired in the UV (DAPI) and green fluorescent protein (GFP) channels after $12 \mathrm{~h}$. A CNT ruleset was applied that detected the whole embryo by auto-fluorescence (UV, GFP) and the transgene as a lower hierarchy sub-object (GFP, CNT applied), permitting transgene-specific measurements of intensity and shape. UPHD25 increases the Lhxla-EGFP expression and causes characteristic changes in transgene morphology. Lower panel: GFP channel fluorescence micrographs in lateral view of $48 \mathrm{hpf}$ $\mathrm{Tg}$ (Cdh17:EGFP) $)^{p t 305}$, treated with vehicle or UPHD25 (800 nM). for therapeutic intervention across a range of different AKI etiologies that may be given to patients late, after they develop established clinical signs of AKI.

\section{Developing an Integrated Phenotypic and Target-Based Screen for Lead Optimization}

Knowledge of molecular targets helps medicinal chemistry campaigns for lead optimization by guiding structure activity relationship studies [12]. A variety of approaches ranging from serendipity to affinity capture proteomics can be used to identify drug targets [26]. Forward genetic approaches using new genome-editing techniques can also be performed in zebrafish and used for rapid target validation [27]. PTBA, UPHD25, and 186 are all HDAC inhibitors (HDIs) [18, 25], and other HDIs, such as Trichostatin $\mathrm{A}$, are active in EKPC assays [18]. However, the HDI, MS275, has no effect in EKPC assays, or on zebrafish larval survival after AKI, and does not prevent post-injury fibrosis after IR-AKI (online suppl. Fig. S1). These data suggest that while HDACs might be a target of PTBA analogs, their MOA requires multiple targets or is dependent on unique HDAC selectivity. Our lab is using a variety of chemical and genetic approaches to test this hypothesis in order to develop an iterative target and phenotype-driven approach for PTBA analog optimization (online suppl. Fig. 2). We are combining this with early pharmaceutical profiling, and screening through a series of secondary AKI assays, including the use of more complex mouse AKI models that reflect the diversity of injury and comorbidities seen in clinical practice, as well as human IPS cell-derived kidney organoids to evaluate effects on toxin-induced AKI [28]. In this way, we hope to identify new PTBA-like chemotypes with unique and favorable efficacy profiles (including data on their potential to modify human kidney cell responses to injury) and structural and pharmaceutical properties for pre-clinical development.

The zebrafish embryonic kidney appears as a pair of tubular structures that converge at the cloaca (triangle). A CNT ruleset was applied that detected the fluorescent kidney (orange). The cloaca was classified based on the brightness and proximity to the zebrafish edge (blue), permitting quantification of Cdh17-EGFP in a defined length tubular segment (red). UPHD25 increases GFP intensity within the embryonic zebrafish kidney. For all panels, rostral is to the left, dorsal pointing up. b Dose-response curves of UPHD25 on kidney progenitor cell expansion using Lhxla-EGFP and kidney organ size by EGFP-Cdh17 with similar EC50 values for UPHD25 in the 2 models.

(For figure see next page.) 


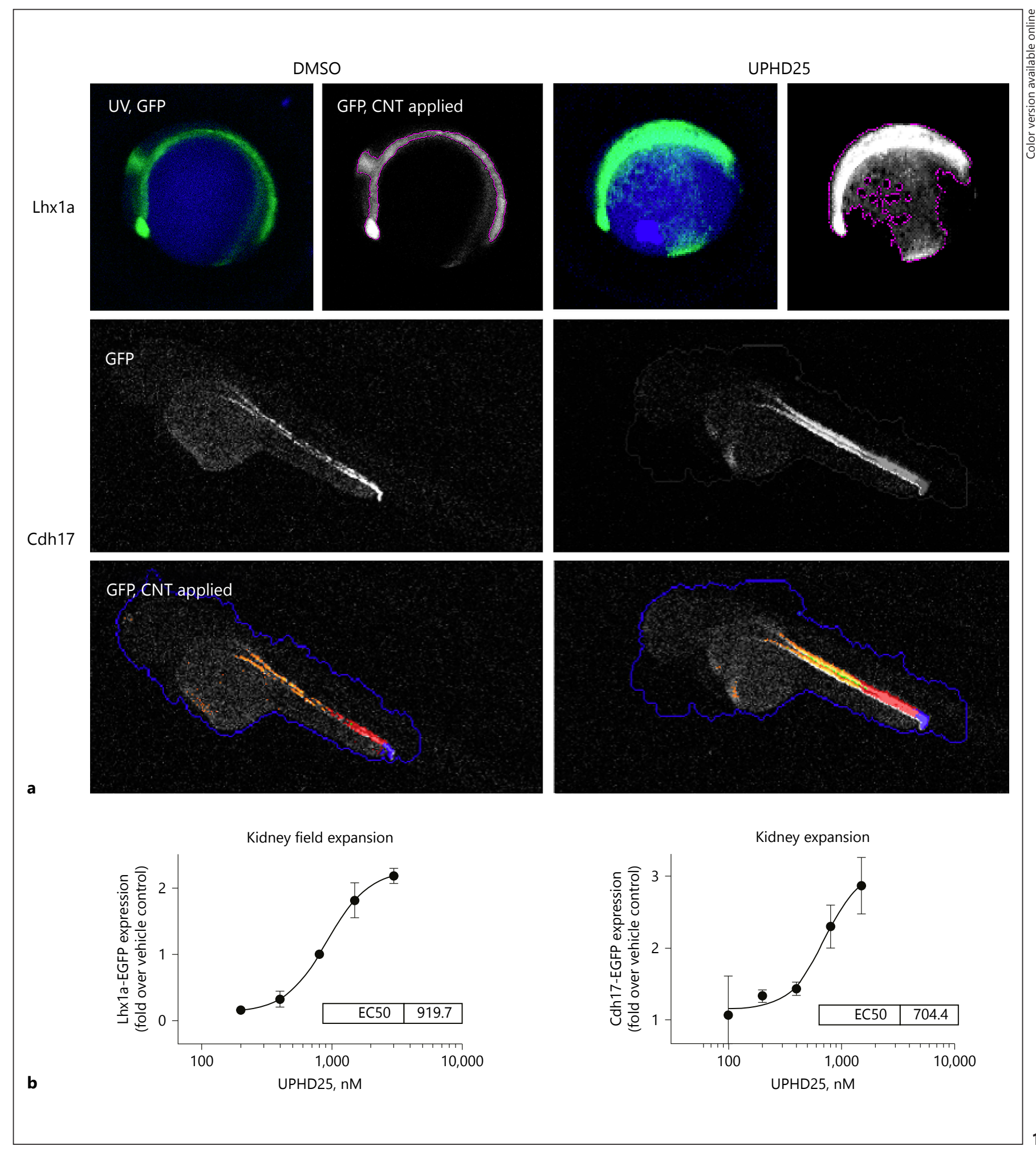




\section{Acknowledgments}

The laboratory of Mark de Caestecker was supported by National Institutes of Health Grants 1RC4DK090770; RO1DK06940305S1 and U24 DK076169; laboratory of Neil Hukriede was supported by the National Institutes of Health Grants R01 DK069403; R01 DK112652; R01 HD053287 and P30 DK079307 for this work. These studies also used the UPCI Chemical Biology Core Facility, which is supported by P30CA047904.

\section{Disclosure Statement}

The authors have no conflicts of interest to declare.

\section{References}

1 Coca SG, Singanamala S, Parikh CR: Chronic kidney disease after acute kidney injury: a systematic review and meta-analysis. Kidney Int 2012;81:442-448.

2 Lo LJ, Go AS, Chertow GM, et al: Dialysisrequiring acute renal failure increases the risk of progressive chronic kidney disease. Kidney Int 2009;76:893-899.

3 Wald R, Quinn RR, Luo J, et al: Chronic dialysis and death among survivors of acute kidney injury requiring dialysis. JAMA 2009;302: 1179-1185.

4 Go AS, Chertow GM, Fan D, McCulloch CE, Hsu CY: Chronic kidney disease and the risks of death, cardiovascular events, and hospitalization. N Engl J Med 2004;351:1296-1305.

5 Faubel S, Chawla LS, Chertow GM, Goldstein SL, Jaber BL, Liu KD: Ongoing clinical trials in AKI. Clin J Am Soc Nephrol 2012;7:861873.

6 Skrypnyk NI, Siskind LJ, Faubel S, de Caestecker MP: Bridging translation for acute kidney injury with better preclinical modeling of human disease. Am J Physiol Renal Physiol 2016;310:F972-F984.

7 Humphreys BD, Cantaluppi V, Portilla D, et al: Targeting endogenous repair pathways after AKI. J Am Soc Nephrol 2016;27:990-998.

8 Swinney DC, Anthony J: How were new medicines discovered? Nat Rev Drug Discov 2011; 10:507-519.

9 Okusa MD, Rosner MH, Kellum JA, Ronco C; Acute Dialysis Quality Initiative XIII Workgroup: Therapeutic targets of human AKI: harmonizing human and animal AKI. J Am Soc Nephrol 2016;27:44-48.
10 Strange K: Drug discovery in fish, flies, and worms. ILAR J 2016;57:133-143.

11 Giacomotto J, Segalat L: High-throughput screening and small animal models, where are we? Br J Pharmacol 2010;160:204-216.

12 Lee JA, Berg EL: Neoclassic drug discovery: the case for lead generation using phenotypic and functional approaches. J Biomol Screen 2013;18:1143-1155.

13 MacRae CA, Peterson RT: Zebrafish as tools for drug discovery. Nat Rev Drug Discov 2015;14:721-731

14 Detrich HW 3rd, Westerfield M, Zon LI: Overview of the zebrafish system. Methods Cell Biol 1999;59:3-10.

15 Williams CH, Hong CC: Zebrafish small molecule screens: taking the phenotypic plunge. Comput Struct Biotechnol J 2016;14:350356.

16 Wingert RA, Davidson AJ: The zebrafish pronephros: a model to study nephron segmentation. Kidney Int 2008;73:1120-1127.

17 Swanhart LM, Cosentino CC, Diep CQ, Davidson AJ, de Caestecker M, Hukriede NA: Zebrafish kidney development: basic science to translational research. Birth Defects Res C Embryo Today 2011;93:141-156.

18 de Groh ED, Swanhart LM, Cosentino CC, et al: Inhibition of histone deacetylase expands the renal progenitor cell population. J Am Soc Nephrol 2010;21:794-802.

19 Cianciolo Cosentino C, Roman BL, Drummond IA, Hukriede NA: Intravenous microinjections of zebrafish larvae to study acute kidney injury. J Vis Exp 2010;42:pii:2079.

20 Cianciolo Cosentino C, Skrypnyk NI, Brilli LL, et al: Histone deacetylase inhibitor enhances recovery after AKI. J Am Soc Nephrol 2013;24:943-953.
21 Skrypnyk NI, Harris RC, de Caestecker MP: Ischemia-reperfusion model of acute kidney injury and post injury fibrosis in mice. J Vis Exp 2013;78.

22 Novitskaya T, McDermott L, Zhang KX, et al: A PTBA small molecule enhances recovery and reduces postinjury fibrosis after aristolochic acid-induced kidney injury. Am J Physiol Renal Physiol 2014;306:F496-F504.

23 Vogt A, Cholewinski A, Shen X, et al: Automated image-based phenotypic analysis in zebrafish embryos. Dev Dyn 2009;238:656-663.

24 Sanker S, Cirio MC, Vollmer LL, et al: Development of high-content assays for kidney progenitor cell expansion in transgenic zebrafish. J Biomol Screen 2013;18:1193-1202.

25 Skrypnyk NI, Sanker S, Skvarca LB, et al: Delayed treatment with PTBA analogs reduces postinjury renal fibrosis after kidney injury. Am J Physiol Renal Physiol 2016;310:F705F716.

26 Schenone M, Dancik V, Wagner BK, Clemons PA: Target identification and mechanism of action in chemical biology and drug discovery. Nat Chem Biol 2013;9:232-240.

27 Schenk H, Muller-Deile J, Kinast M, Schiffer M: Disease modeling in genetic kidney diseases: zebrafish. Cell Tissue Res 2017, Epub ahead of print.

28 Morizane R, Lam AQ, Freedman BS, Kishi S, Valerius MT, Bonventre JV: Nephron organoids derived from human pluripotent stem cells model kidney development and injury. Nat Biotechnol 2015;33:1193-1200. 